\title{
Measuring mass and angular momentum of black holes with high-frequency quasi-periodic oscillations
}

\author{
B. Aschenbach
}

\author{
Max-Planck-Institut für extraterrestrische Physik, PO Box 1312, Garching bei München, 85741, Germany \\ e-mail: bra@mpe.mpg.de
}

Received 4 June 2004 / Accepted 1 July 2004

\begin{abstract}
For the three microquasars GRO J1655-40, XTE J1550-564 and GRS 1915+105 twin high frequency quasi-periodic oscillations (HFQPOs) with a ratio of 3:2 and/or 3:1 have been measured. For a test particle orbiting a rotating black hole on a stable circular orbit there exist two different orbits at which the vertical and radial epicyclic oscillations are in either a 3:1 or 3:2 parametric resonance for any choice of the black hole angular momentum $a$. If the two orbits are required to be frequency commensurable Keplerian orbits there is only one solution for the two orbit radii and $a$. This model predicts that the microquasars have the same $a$, and it predicts their black hole masses on the basis of the measured HFQPOs in agreement with the dynamically determined masses. Application of this model to the Galactic Center black hole Sgr A* using the recently measured QPOs (Genzel et al. 2003; Aschenbach et al. 2004) leads to a black hole mass of $(3.28 \pm 0.13) \times 10^{6} M_{\odot}$, and the same $a$ as for the microquasars. The possibility that all four sources have $a=0.99616$ suggests that this value is the upper limit of $a$ imposed by general relativity. The same value for the lower orbit radius and the same value for $a$ are also suggested by an analysis of the general relativistic expression for the radial gradient of the orbital velocity, which changes sign in a narrow annular region around the lower orbit when $a>0.9953$.
\end{abstract}

Key words. accretion, accretion discs - relativity - Galaxy: center - Galaxy: fundamental parameters - X-rays: general black hole physics

\section{Introduction}

High-frequency qasi-periodic oscillations (HFQPO) have been observed in quite a number of X-ray binaries containing a compact object, i.e. neutron stars or black holes. HFQPOs are considered to originate in the accretion disk around the compact object. A special group is obviously set up by the galactic microquasars GRO J1655-40, XTE J1550-564 and GRS $1915+105$. They are special because they do not show just one frequency but twin frequencies at a fixed ratio of $3: 2$ (Abramowicz \& Kluźniak 2001; Miller et al. 2001; Remillard et al. 2002), which are 450, $300 \mathrm{~Hz}$ (Strohmayer 2001a; Remillard et al. 1999), 276, $184 \mathrm{~Hz}$ (Miller et al. 2001; Remillard et al. 2002) and 168, $113 \mathrm{~Hz}$ (Remillard et al. 2003; McClintock \& Remillard 2004), respectively. Out of those three objects XTE J1550-564 might have an additional power spectral density peak at $92 \mathrm{~Hz}$, the significance of which is modest, however (Remillard et al. 2002).

Around the time the discoveries were made, it was suggested independently that some resonance effect in the accretion disk could give rise to such a 3:2 frequency behaviour (Kluźniak \& Abramowicz 2001a,b). There are three fundamental oscillation modes for a test particle orbiting a black hole, which produce the orbital or azimuthal (Kepler) frequency, the radial (in the orbital plane) epicyclic frequency and the vertical or polar (perpendicular to the orbital plane) epicyclic frequency. Initially it has been suggested that the 3:2 frequency ratio is due to a coupling of orbital and radial frequency (Abramowicz \& Kluźniak 2001; Schnittman \& Bertschinger 2004). However, the proponents of the resonance theory then concentrated on a parametric 3:2 resonance between the polar and the radial epicyclic modes (Kluźniak \& Abramowicz 2002; Abramowicz et al. 2002, 2003; Abramowicz \& Kluźniak 2003; Kluźniak \& Abramowicz 2003; Rebusco 2004), and Remillard et al. (2002) found solutions for a 3:2 frequency ratio for each of the three possible couplings, i.e. orbital/radial, orbital/polar and radial/polar. The method of associating a frequency to one specific oscillation mode has in principle the benefit that from frequency measurements the mass $M$, the angular momentum $a$ (the Kerr parameter) of the black hole and the radial position of the orbiting test particle in the accretion disk can be determined. But with the measurement of even several frequencies the relevant equations remain underdetermined and more information is needed. So, in general the mass, which has been determined from the dynamics of the binary orbit for each of the three microquasars, has been made use of and a value of $a$ could be determined, which, however, is different for each of the three coupling possibilities. Furthermore, as Remillard et al. (2002) have pointed out on their findings on XTE J1550-564 that the frequency ratio might not be just $3: 2$ but that $3: 1$ is another 
possibility, one ends up with a choice for $a$ of one out of six, still adopting $M$ from dynamical measurements. There may be a possibility to resolve this ambiguity by introducing another constraint in the resonance model.

I have investigated whether both a $3: 2$ and a $3: 1$ parametric resonance between the vertical and radial epicyclic frequencies would exist, of course at two different orbits, but that these two orbits are commensurable orbits. The commensurability is meant in the traditional sense that the Kepler frequencies of the two orbits come in ratios of natural numbers. There does indeed exist only one such configuration, so that the Kepler frequency of the inner orbit is three times the Kepler frequency of the outer orbit. Furthermore, this solution allows only one value for the angular momentum, which is $a=0.99616$. The mass of the black hole $M$ is uniquely determined after a choice for one of the two possible orbits has been made. It is likely that the discriminator between the orbits is the mass accretion rate. In any case the two possible values for $M$ differ by a factor of 1.5 only. The masses of the three black holes in GRO J1655-40, XTE J1550-564 and GRS 1915+105, predicted by this model on the basis of their measured HFQPOs, agree in each case with the dynamically determined masses within their measurement uncertainty range. I have applied this model also to the Galactic Center black hole, Sgr A*, making use of the one quasi-period recently published by Genzel et al. (2003) and the quasi-periods published by Aschenbach et al. (2004). Again, the predicted black hole mass agrees very well with the dynamically determined mass (Sect. 3 ). The possible relevance of the 3:2 resonance model to the periodicities reported for Sgr A* has been mentioned in the papers of Kluźniak et al. (2003) and Abramowicz et al. (2004b).

I have checked the general relativistic expressions for the motion of a test particle orbiting a rotating black hole using the canonical Boyer-Lindquist functions for some unexpected behaviour near the two orbits and in the angular momentum range around $a=0.99616$. Energy and angular momentum of the test particle remain unconspicuous, but the orbital velocity does not. Whereas for any $a<0.9953$ the orbital velocity is monotonically increasing with decreasing radius over the full range of the radius down to the marginally stable orbit, for $a>0.9953$ the orbital velocity starts to decrease with decreasing radius over a small radial range before it again rises with decreasing radius. This annular range of decreasing orbital velocity happens to be located where the 3:1 parametric resonance of the vertical and radial epicyclic frequencies appears for $a=0.99616$. To me it seems that this finding is not a chance coincidence but is further support of the resonance model above. The detailed physics still have to be explored. Further details are given in Sect. 4. Some discussion about the implications is presented in Sect. 5 .

\section{The model}

There are three fundamental cyclic gravitational modes associated with black hole accretion disks (Nowak \& Lehr 1998; Merloni et al. 1999), which are the Kepler frequency $\left(\Omega_{\mathrm{K}}\right)$, the disk perturbation frequencies in vertical and radial direction called vertical $\left(\Omega_{\mathrm{V}}\right)$ and radial $\left(\Omega_{\mathrm{R}}\right)$ epicyclic frequency.

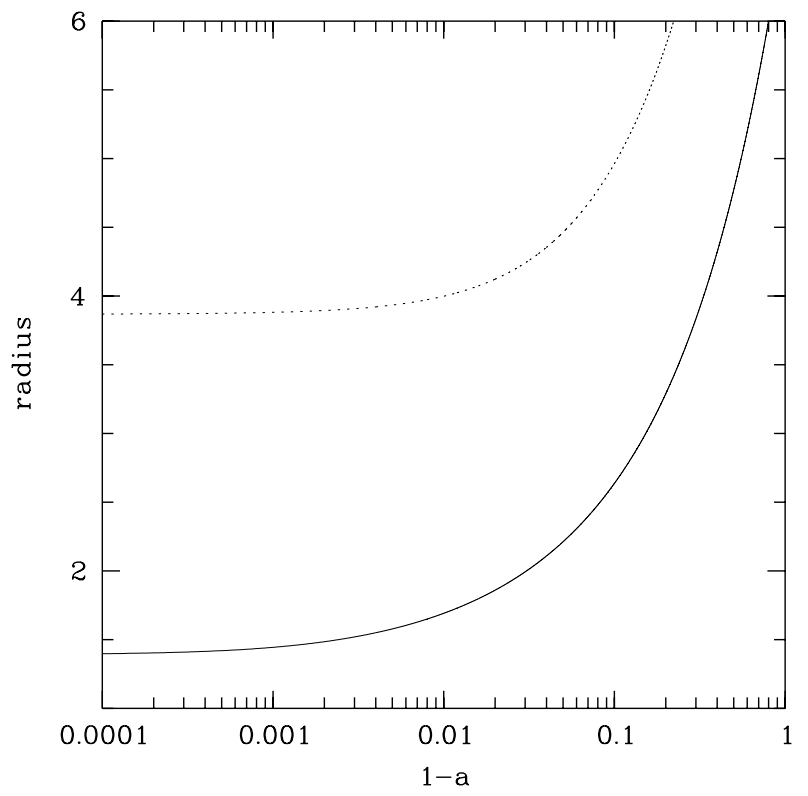

Fig. 1. Radii of orbits for which $\Omega_{\mathrm{V}} / \Omega_{\mathrm{R}}=3: 1$ (solid line) or $\Omega_{\mathrm{V}} / \Omega_{\mathrm{R}}=3: 2$ (dashed line) versus $(1-a)$.

Each frequency depends on the central, black hole mass $M$, its angular momentum $a$ and the radial distance $r$ from the center. Equations (1) to (3) show the relations, for which the standard notation of $c=G=1$ is used. Physical length scales are in units of $G M / c^{2}$ and angular frequencies $\Omega$ are in units of $c^{3} / G M . r=1$ is defined as the gravitational radius $r_{\mathrm{g}}$. The conversion from observed linear frequency $v$ measured in $\mathrm{Hz}$ to the normalized $\Omega$ is obtained through $v=\Omega \times c^{3} /(2 \pi G M)$ with $c$, $G, M$ in cgs units.

$\Omega_{\mathrm{K}}=\left(r^{3 / 2}+a\right)^{-1}$

$\Omega_{\mathrm{V}}^{2}=\Omega_{\mathrm{K}}^{2}\left(1-\frac{4 a}{r^{3 / 2}}+\frac{3 a^{2}}{r^{2}}\right)$

$\Omega_{\mathrm{R}}^{2}=\Omega_{\mathrm{K}}^{2}\left(1-\frac{6}{r}+\frac{8 a}{r^{3 / 2}}-\frac{3 a^{2}}{r^{2}}\right)$.

The model I propose to explain the twin HFQPOs observed at a ratio of $3: 2$ or $3: 1$ should have both $\Omega_{\mathrm{V}} / \Omega_{\mathrm{R}}=3: 2$ and $\Omega_{\mathrm{V}} / \Omega_{\mathrm{R}}=3: 1$ for the same angular momentum $a$. Of course, this requires two different orbits with different radii called $r_{32}$ and $r_{31}$, which follow from Eqs. (1) to (3). Figure 1 shows $r_{32}$ and $r_{31}$ versus $(1-a)$. There is no limitation on $a$, and $r_{32}$ and $r_{31}$ exist for any $a$. The second requirement is that the two orbits should be commensurable orbits, which in traditional sense means that $\Omega_{\mathrm{K}}\left(r=r_{31}\right)=n \times \Omega_{\mathrm{K}}\left(r=r_{32}\right)$ in it's simplest form, with $n$ a natural number. Figure 2 shows $\Omega_{\mathrm{K}}\left(r=r_{31}, a\right) / \Omega_{\mathrm{K}}\left(r=r_{32}, a\right)$ versus $(1-a)$. The maximum value of this ratio is 3.26 and the minimum value is 2.026 . Therefore there is a solution but only one solution for $n=3$, which in turn fixes the values of $a$ to $a=0.99616, r_{31}=1.546$ and $r_{32}=3.919$. Values of $a, r_{31}$ and $r_{32}$ are truncated.

Table 1 summarizes the values of the relevant frequencies $\Omega$ including the Lense-Thirring precession frequency 


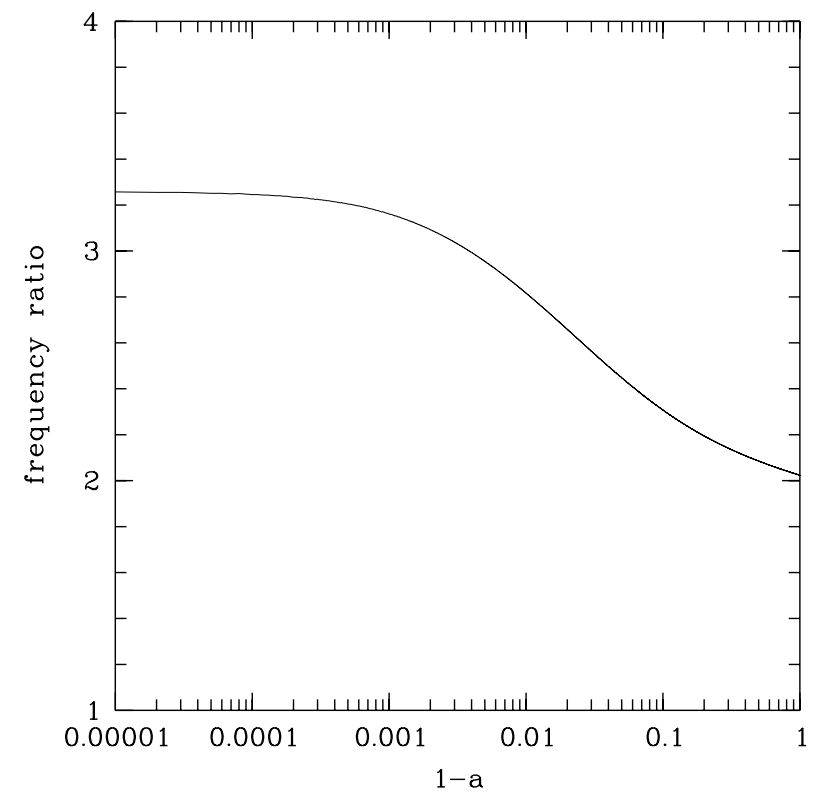

Fig. 2. Ratio of Kepler frequencies $\Omega_{\mathrm{K}}\left(r=r_{31}, a\right) / \Omega_{\mathrm{K}}\left(r=r_{32}, a\right)$ versus $(1-a)$.

Table 1. Compilation of frequency values for $a=0.99616, r_{31}=$ 1.546 and $r_{32}=3.919$.

\begin{tabular}{ccc}
\hline \hline$\Omega_{31}$ & $r_{32}$ \\
\hline$\Omega_{\mathrm{K}}$ & 0.342684 & 0.114228 \\
$\Omega_{\mathrm{V}}$ & 0.142370 & 0.0942112 \\
$\Omega_{\mathrm{R}}$ & 0.0475116 & 0.0628118 \\
$\Omega_{\mathrm{b}}$ & 0.0948583 & 0.0313993 \\
$\Omega_{\mathrm{LT}}$ & 0.200313 & 0.0200171 \\
\hline
\end{tabular}

$\Omega_{\mathrm{LT}}=\Omega_{\mathrm{K}}-\Omega_{\mathrm{V}}$ (Merloni et al. 1999) for $\mathrm{r}_{31}$ and $\mathrm{r}_{32}$. The parametric resonances are for ratios of $3: 1$ and 3:2. I note that if a beat frequency of $\Omega_{\mathrm{b}}=\Omega_{\mathrm{V}}-\Omega_{\mathrm{R}}$ exists, which may be indicated by the observation of the $92 \mathrm{~Hz}$ frequency in XTE J1550-564, then the parametric resonances would be a 3:2:1 triplet both at $r_{31}$ and $r_{32}$. Therefore I include $\Omega_{\mathrm{b}}$ in Table 1 .

It is evident from Table 1 that the highest frequency involved in both the 3:2 and 3:1 resonance is $\Omega_{\mathrm{V}}$. Accordingly, the black hole mass can be calculated for the $3: 1$ resonance $\left(M_{31}\right)$ and the 3:2 resonance $\left(M_{32}\right)$ from Eqs. (4) and (5) based on the HFQPO measured uppermost frequency $v_{\text {up }}$ in $\mathrm{Hz}$ :

$$
\begin{aligned}
& M_{31} / M_{\odot}=4603.3 / v_{\text {up }} \\
& M_{32} / M_{\odot}=3046.2 / v_{\text {up }} .
\end{aligned}
$$

Equations (4) and (5) illustrate that $M_{31}$ and $M_{32}$ differ by factor of 1.51 only.

Apart from the parametric resonances enforced by the initial requirements, i.e. $\Omega_{\mathrm{V}}\left(r=r_{31}\right) / \Omega_{\mathrm{R}}\left(r=r_{31}\right)=3: 1$, $\Omega_{\mathrm{V}}\left(r=r_{32}\right) / \Omega_{\mathrm{R}}\left(r=r_{32}\right)=3: 2$ and $\Omega_{\mathrm{K}}\left(r=r_{31}\right) / \Omega_{\mathrm{K}}(r=$ $\left.r_{32}\right)=3: 1$ it turns out that there are "cross" resonances connecting $r_{31}$ and $r_{32}$, which are, apart from the Kepler commensurable orbits, $\Omega_{\mathrm{R}}\left(r=r_{32}\right) / \Omega_{\mathrm{R}}\left(r=r_{31}\right)=4: 3$ and
$\Omega_{\mathrm{V}}\left(r=r_{32}\right) / \Omega_{\mathrm{R}}\left(r=r_{31}\right)=2: 1$ (cf. Table 1 ). In summary, $r_{31}$ and $r_{32}$ show an extraordinary high degree of parametric resonances.

With $a=0.99616$ some radii, useful to know, can be calculated. The event horizon is at $r_{\mathrm{H}}=1.088$; the static limit in the equatorial plane is at $r_{0}=2$ and the marginally stable orbit is at $r_{\mathrm{ms}}=1.306$ (defined by $\Omega_{\mathrm{R}}=0$; Bardeen et al. 1972), which means that both $r_{31}$ and $r_{32}$ are stable orbits, and that $r_{31}$ is within the ergosphere whereas $r_{32}$ is outside of it. In fact, $r_{31}=\left(r_{\mathrm{H}}+r_{0}\right) / 2$, which is mid-way between the event horizon and the static limit in the equatorial plane.

\section{Comparison with observations}

The model so far has made use of just frequency ratios not so much of the absolute frequencies. The test of the model would consist of a comparison with otherwise measured masses or angular momenta. For the three microquasars GRO J1655-40, XTE J1550-564 and GRS 1915+105 measurements of their masses have been made but there is so far no way to determine the angular momenta, so the test is necessarily restricted to their masses. Masses are usually derived from the determination of the orbit parameters in case of a binary. This holds for the three microquasars but also for the black hole Sgr A* in the Galactic Center which is orbited by the S2 (S0-2) star (Schödel et al. 2002; Ghez et al. 2003). Genzel et al. (2003) have reported a QPO period of $16.8 \mathrm{~min}$ observed in two nearinfrared flares. Aschenbach et al. (2004) have claimed additionally quasi-periods around $219 \mathrm{~s}, 700 \mathrm{~s}, 1150 \mathrm{~s}$ and $2250 \mathrm{~s}$, with the $1150 \mathrm{~s}$ period being consistent with the NIR period. This set of quasi-periods was found in the power density spectra of one X-ray flare observed with Chandra (Baganoff et al. 2001) and a second X-ray flare observed with XMM-Newton (Porquet et al. 2003). Interesting in this context is that the frequencies corresponding to the latter three quasi-periods are close to a 3:2:1 ratio. Enforcing such a ratio sequence in a best fit, it turns out that such a ratio is consistent with the measurements. Accordingly, I include the Galactic Center black hole in this test and predict the mass of Sgr A*.

\subsection{GRO J1655-40}

The frequencies in question are 450 and $300 \mathrm{~Hz}$ (Strohmayer 2001a; Remillard et al. 1999) with $\Omega_{\mathrm{V}}=450 \mathrm{~Hz}$. According to Eq. (5) the predicted black hole mass is $M_{\mathrm{BH}}=6.76 \pm 0.1 M_{\odot}$. The relative uncertainty of the mass is the same as that of the HFQPO measurements of $\approx 1.5 \%$. Dynamical mass measurements have been reported by Orosz \& Bailyn (1997) with $M_{\mathrm{BH}}=7.02 \pm 0.22 M_{\odot}$ and more recently by Greene et al. (2001) who obtained a mass range of $M_{\mathrm{BH}}=5.8-6.8 M_{\odot}$. The agreement between model prediction and observation is quite satisfactory.

\subsection{XTE J1550-564}

Frequencies around 276 and $184 \mathrm{~Hz}$ (Miller et al. 2001; Remillard et al. 2002) have been measured. With $\Omega_{\mathrm{V}}=276 \mathrm{~Hz}$ Eq. (5) predicts a black hole mass of $M_{\mathrm{BH}}=11.04 \pm 0.2 M_{\odot}$. 
The relative uncertainty of the mass is again due to the accuracy of the HFQPO measurements of $\approx 2 \%$. Dynamical mass measurements have been reported by Orosz et al. (2002a) with a $\pm 1 \sigma$ mass range of $M_{\mathrm{BH}}=8.4-11.6 M_{\odot}$, which is nicely matched by the predicted mass.

\subsection{GRS $1915+105$}

The relevant 3:2 pair at 168 and $113 \mathrm{~Hz}$ has only recently been found (Remillard et al. 2003; McClintock \& Remillard 2004). With $\Omega_{\mathrm{V}}=168 \mathrm{~Hz}$ Eq. (5) predicts a black hole mass of $M_{\mathrm{BH}}=18.13 \pm 0.36 M_{\odot}$. The relative uncertainty of the mass is due the accuracy of the HFQPO measurements of $\approx 2 \%$. Dynamical mass measurements have been reported by Greiner at al. (2001) with $M_{\mathrm{BH}}=14.0 \pm 4.0 M_{\odot}$ and Harlaftis \& Greiner (2004) with $M_{\mathrm{BH}}=14.0 \pm 4.4 M_{\odot}$. Also in this case the predicted mass matches the dynamically mass within the $\pm 1 \sigma$ range, but the measurements of this source illustrate nicely the potential uncertainty in dynamical mass measurements. Harlaftis \& Greiner (2004) point out that the biggest uncertainty is related to the inclination of the orbit, which for GRS $1915+105$ is taken from the inclination of the associated jet assuming that the jet axis is orthogonal to the orbital plane of the binary. If, for instance, the inclination angle is changed from the adopted $66^{\circ}$ to $56^{\circ}$ the estimated black hole mass is $M_{\mathrm{BH}}=16.9 \pm 5.9 M_{\odot}$ (Harlaftis \& Greiner 2004). Actually, Kaiser et al. (2004) suggest that the inclination angle is $53^{\circ}$ putting the best estimate for the dynamical black hole mass at $17.8 M_{\odot}$, which is within the $\pm 1 \sigma$ measurement of Harlaftis $\&$ Greiner (2004), but it is also close to the predicted value of $18.1 M_{\odot}$.

\subsection{XTE J1859-226}

Cui et al. (2000) report the measurement of a single HFQPO at $187 \mathrm{~Hz}$ associated with XTE J1859-226. Filippenko \& Chornock (2001) announced a mass function of the binary of $7.4 M_{\odot}$, and Brocksopp et al. (2002) discovered associated radio jets suggesting that XTE J1859-226 is another galactic microquasar. Orosz et al. (2002b) and McClintock \& Remillard (2004) finally quote an estimated mass of the black hole between 7.6 and $12.0 M_{\odot}$. So far there is just one HFQPO measured so that a prediction based on the proposed model is difficult, but there are only two options. If the $187 \mathrm{~Hz}$ frequency corresponds to the vertical epicyclic 3:2 resonance the predicted mass (Eq. (5)) is about $16.3 M_{\odot}$ and a second HFQPO at $125 \mathrm{~Hz}$, i.e. the radial epicyclic frequency, should be looked for. If, on the contrary, the $187 \mathrm{~Hz}$ frequency is the result of the radial epicyclic frequency the expected vertical epicyclic frequency is at $281 \mathrm{~Hz}$ and the predicted mass is about $10.9 M_{\odot}$, which fits quite nicely in the mass range given by Orosz et al. (2002b) and McClintock \& Remillard (2004), but these authors note that the mass range is quite uncertain. A deep search for a second HFQPO would be extremely useful.

\subsection{The Galactic Center black hole Sgr A*}

Aschenbach et al. (2004) have grouped the frequencies indicated in the power density spectra of two NIR flares (Genzel et al. 2003) and two X-ray flares. There are three groups for which there is evidence that each of the frequencies of one group occurred in at least one NIR and both X-ray flares. These three groups of quasi-periods in seconds, ordered by NIR, Chandra, XMM-Newton, are group 1: $(733,692,701)$, group 2: $(1026,1117,1173)$ and group $3:(-, 2307,2178)$; the duration of the NIR flare was too short to cover the longest period. Looking at the average quasi-period within one group it appears as if the ratio of the average quasi-periods follows a $1: 1.5: 3$ relation which is equivalent to a $3: 2: 1$ relation in frequencies. This is at least consistent in view of the frequency measurement uncertainties. Each of these eight quasi-periods has been observed at low wave number which means that the systematic uncertainty of each period determined from the associated power density spectra is up to $\pm 20 \%$. Enforcing a 3:2:1 ratio sequence in a best fit the highest frequency is $v_{\text {up }}=1.4035 \pm 0.0574 \mathrm{mHz}$ or a period of $712 \pm 29 \mathrm{~s}$. The two remaining quasi-periods are at $1069 \pm 44 \mathrm{~s}$ and $2138 \pm 87 \mathrm{~s}$. In the paper of Aschenbach et al. (2004) we associated the quasiperiod of group 1 with the vertical epicyclic oscillation at the marginally stable orbit and the quasi-period of group 2 with the radial epicyclic oscillation at the radius at which the radial oscillations have their maximum frequency. This together with the association of the $219 \mathrm{~s}$ quasi-period to the Kepler period of the marginally stable orbit led to a unique value for the mass and the angular momentum of the black hole. For the quasiperiod of group 3 (2150 s) we had no convincing suggestion. This together with the fact that we needed to employ different radial positions for the different frequencies is unsatisfactory. This is taken care now by the association of the quasi-periods of group 1, group 2 and group 3 with the epicyclic modes at just one radius.

Unlike the microquasars which show two frequencies Sgr A* appears to show three frequencies of about similar power spectral densities (Aschenbach et al. 2004), so that it makes sense to classify this as an example of the $3: 1$ resonance for which Eq. (4) applies, and the black hole mass of Sgr A* is predicted to $M_{\mathrm{BH}}=(3.28 \pm 0.13) \times 10^{6} M_{\odot}$. Measurements of the orbital motion of the star S0-2 (S2) around the GC black hole have resulted in $M_{\mathrm{BH}}=(3.7 \pm 1.5) \times 10^{6} M_{\odot}$ (Schödel et al. 2002), $M_{\mathrm{BH}}=(4.07 \pm 0.62) \times 10^{6} M_{\odot}\left(R_{0} / 8 \mathrm{kpc}\right)^{3}$ (Ghez et al. 2003) with $R_{0}$ the distance to the black hole, and $M_{\mathrm{BH}}=(3.59 \pm 0.59) \times 10^{6} M_{\odot}$ (Eisenhauer et al. 2003). Errors quoted for each of the three mass measurements are $1 \sigma$. The mass predicted by the present model agrees with both the Schödel et al. (2002) mass and the mass given by Eisenhauer et al. (2003) but it is slightly out of the $1 \sigma$ range of the Ghez et al. (2003) measurement for $R=8 \mathrm{kpc}$.

Application of Eq. (5), checking for a 3:2 resonance, gives $M_{\mathrm{BH}}=(2.17 \pm 0.1) \times 10^{6} M_{\odot}$, which is ruled out by the dynamical measurements. This supports that in the framework of this model the accretion disk around $\operatorname{Sgr} \mathrm{A}^{*}$ is in a 3:1 resonance. The third frequency associated with the resonance state 2 may be either a beat frequency, i.e. $\Omega_{\mathrm{V}}-\Omega_{\mathrm{R}}$ or the first harmonic 
of $\Omega_{R}$. In either case a fourth frequency could be expected which is either $\Omega_{\mathrm{V}}+\Omega_{\mathrm{R}}$ or the third harmonic of $\Omega_{\mathrm{R}}$. This would correspond to resonance state 4 , and the expected period is $534 \pm 22 \mathrm{~s}$. Such a period seems to be present in the power density spectrum of the Chandra flare at $494 \pm 41 \mathrm{~s}$ and in the NIR flare of June 15, 2003 at $498 \pm 100 \mathrm{~s}$. It is absent in the NIR flare of June 16, 2003 and the XMM-Newton flare (Aschenbach et al. 2004). With $a$ and $M_{\mathrm{BH}}$ given the orbital (Kepler) period is $P_{K}\left(r=r_{31}\right)=296 \pm 12 \mathrm{~s}$; at the marginally stable orbit $P_{K}\left(r=r_{\mathrm{ms}}\right)=242 \pm 10 \mathrm{~s}$. The Chandra flare shows increased power spectral density at $P_{\text {Chandra }}=256 \pm 10 \mathrm{~s}$ and the $X M M-N e w t o n$ flare has significant power at $P_{X M M}=219 \pm 15 \mathrm{~s}$ (Aschenbach et al. 2004). These data are consistent with the view that the shortest quasi-periods observed in the X-ray flares correspond to Keplerian motion along orbits between $r_{31}$ and $r_{\mathrm{ms}}$, the actual radius might be different from flare to flare, not so are $\Omega_{\mathrm{V}}$ and $\Omega_{\mathrm{R}}$, of course.

\section{A physical mechanism?}

With the distinct values for $a, r_{31}$ and $r_{32}$ given it comes to mind to look for a physical reason why these quantities are special. I checked the general relativistic expressions of energy, angular momentum and orbital frequency for a test particle moving around a rotating black hole on a stable orbit. These expressions (Shapiro \& Teukolsky 1983) change monotonically with $r$ in $(a, r)$ space. But this is not the case for the orbital velocity $v^{(\Phi)}$ described in the ZAMO-frame (the Zero Angular Momentum Observer or Bardeen observer). $v^{(\Phi)}$ is represented by Eq. (6) (Müller \& Camenzind 2004):

$v^{(\Phi)}=\frac{\tilde{\omega}}{\alpha}(\Omega-\omega)$.

The Boyer-Lindquist functions are (e.g., Müller \& Camenzind 2004):

$\alpha=\frac{\rho \sqrt{\Delta}}{\Sigma}$,

$\Delta=r^{2}-2 M r+a^{2}$,

$\rho^{2}=r^{2}+a^{2} \cos ^{2} \theta$,

$\Sigma^{2}=\left(r^{2}+a^{2}\right)^{2}-a^{2} \Delta \sin ^{2} \theta$,

$\tilde{\omega}=\frac{\Sigma}{\rho} \sin \theta$,

$\omega=\frac{2 a M r}{\Sigma^{2}}$,

$\theta$ is the polar angle with $\theta=\pi / 2$ defining the equatorial plane.

As Müller \& Camenzind (2004) state one usually assumes Keplerian rotation for $r \geq r_{\mathrm{ms}}$ and sets $\Omega=\Omega_{\mathrm{K}}$ (Eq. (1)). Setting $\theta=\pi / 2$ and $\Omega=\Omega_{\mathrm{K}}$ I have solved numerically Eq. (6) over the full $(a, r)$ space. For large values of $r$ and small values of $a, v^{(\Phi)}$ behaves as expected from Newtonian mechanics, i.e. $v^{(\Phi)}$ increases monotonically with decreasing $r$. But this monotonic behaviour changes for $a>0.9953$; $v^{(\Phi)}$ develops a maximum at small values of $r$, decreases with decreasing $r$ until

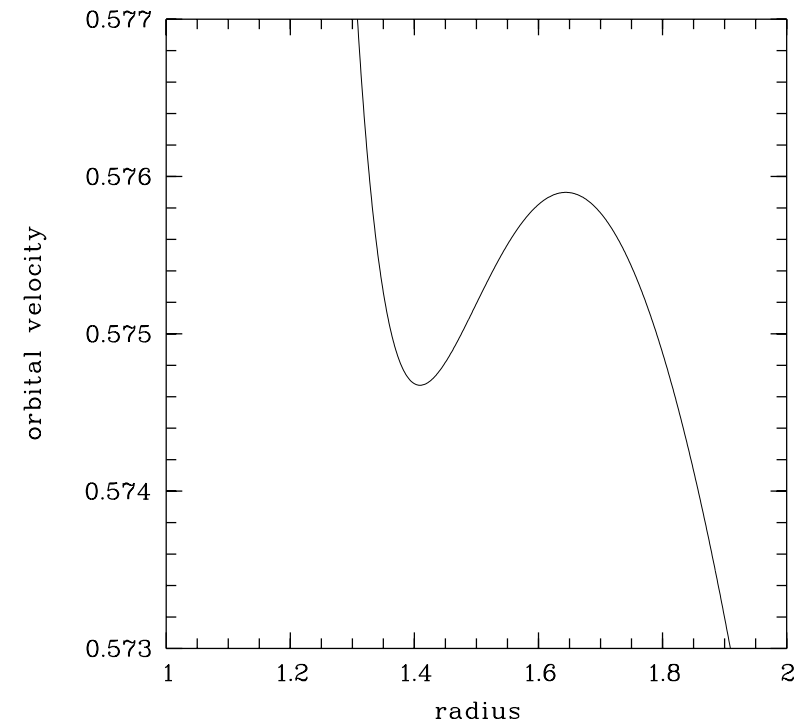

Fig. 3. Orbital velocity $v^{(\Phi)}$ versus orbital radius for $a=0.99616$. Outside the radial range shown $v^{(\Phi)}$ decreases monotonically with radius.

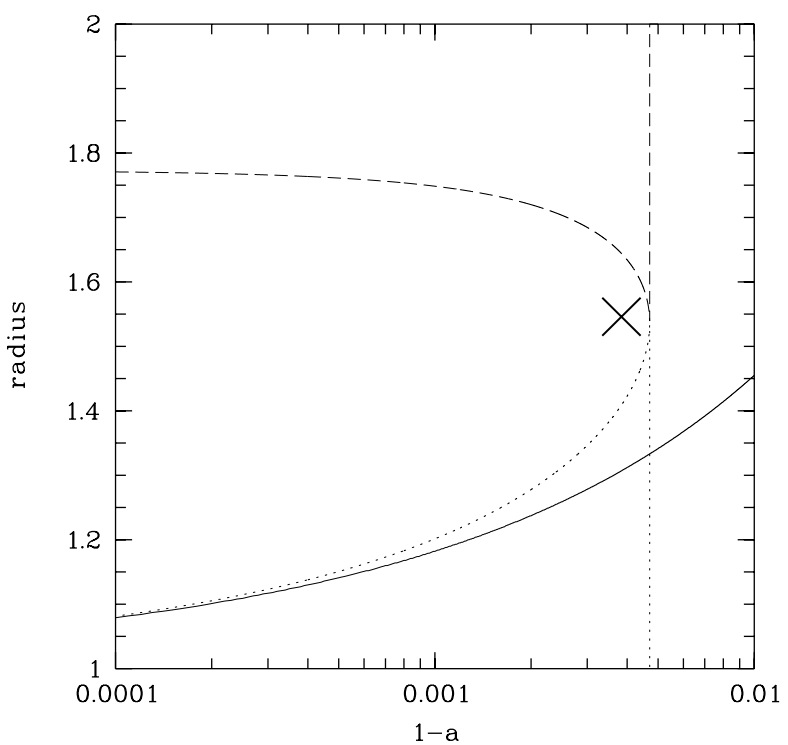

Fig. 4. As a function of $(1-a)$ are shown the radial position $r_{\max }$ of the local maximum of $v^{(\Phi)}$ (curved dashed line), the radial position $r_{\text {min }}$ of the local minimum of $v^{(\Phi)}$ (curved dotted line). The vertical dashed line is at $a=a_{\mathrm{c}}=0.9953$. The cross marks $r_{31}$ and $a=a_{\mathrm{f}}=0.99616$, which is the solution of the parametric resonance model with commensurable orbits. The solid line represents the innermost marginally stable orbit $r_{\mathrm{ms}}$ defined by $\Omega_{\mathrm{R}}=0$.

it reaches a minimum and from thereon increases again with decreasing $r$ until $r_{\mathrm{ms}}$ is reached. Figure 3 shows an example for $a=0.99616$. The depression of $v^{(\Phi)}$ of $0.2 \%$ is small but it increases with increasing $a$. Figure 4 illustrates how the radial position of the local maximum $\left(r_{\max }\right)$ and the local minimum $\left(r_{\min }\right)$ of $v^{(\Phi)}$ change with $a$. The plot also demonstrates that always $r_{\text {min }}>r_{\text {ms }}$. Such a variation with $r$ is absent for the particle energy $E$ and its angular momentum $l$. A main role, that this is different for the orbital velocity, seems to play the appearance of $\omega$, the frame-dragging frequency or potential for 


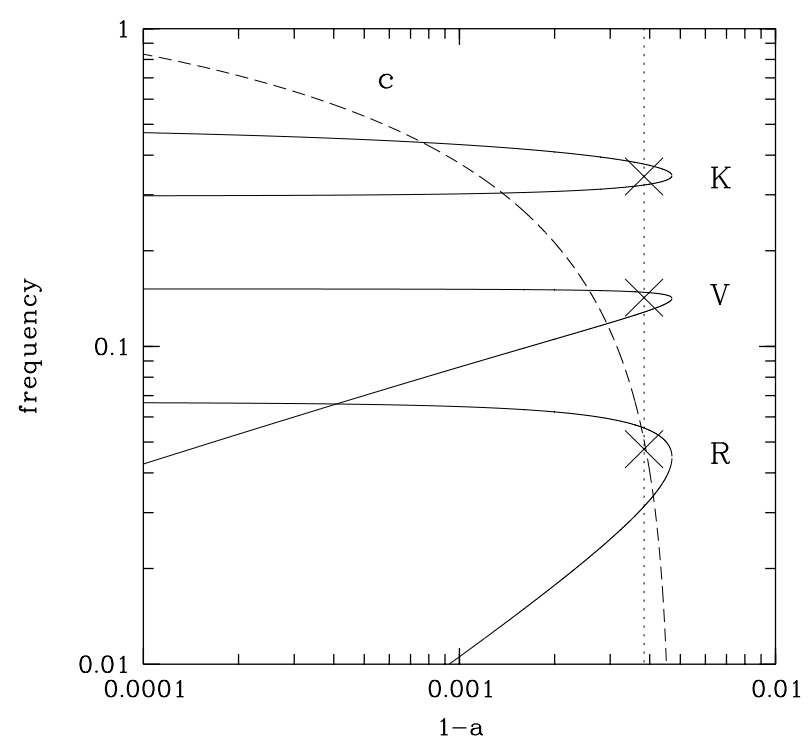

Fig. 5. As a function of $(1-a)$ are shown $\Omega_{\mathrm{K}}\left(r=r_{\min }, a\right)$ and $\Omega_{\mathrm{K}}\left(r=r_{\max }, a\right)$ (label K), which makes up the upper parabola like contour; the contour labeled $\mathrm{V}$ is for $\Omega_{\mathrm{V}}\left(r=r_{\text {min }}, a\right)$ and $\Omega_{\mathrm{V}}\left(r=r_{\max }, a\right)$ and the lowest parabola like contour (R) is for $\Omega_{\mathrm{R}}\left(r=r_{\min }, a\right)$ and $\Omega_{\mathrm{R}}\left(r=r_{\max }, a\right)$. The vertical dashed line is for $a=a_{\mathrm{f}}=0.99616$ and the crosses mark the corresponding frequencies found from the commensurability considerations. Their positions are clearly within the radial section bound by $r_{\min }$ and $r_{\max }$. The dashed line labeled $\mathrm{c}$ represents $\Omega_{\mathrm{c}}=\left.2 \pi \frac{\partial v^{(\Phi)}}{\partial r}\right|_{\max }$.

angular momentum, in Eq. (6). The change of $v^{(\Phi)}$ implies that for $r_{\min } \leq r \leq r_{\max } \partial E / \partial v^{(\Phi)} \leq 0$ and $\partial l / \partial v^{(\Phi)} \geq 0$.

According to standard definition the orbits between $r_{\text {min }}$ and $r_{\max }$ are stable. The region $r_{\min }<r<r_{\max }$ is the only region with $\partial v^{(\Phi)} / \partial r \geq 0$; it also has $\partial^{2} v^{(\Phi)} / \partial r^{2}=0$. The former is basically the rate of change of the orbital velocity per unit length of $r$ seen by the ZAMO. It has the dimension of a frequency. I therefore define an angular "frequency" $\Omega_{\mathrm{c}}=\left.2 \pi \frac{\partial v^{(\Phi)}}{\partial r}\right|_{\max }$, by which the radial position is defined at which $\partial v^{(\Phi)} / \partial r$ has the maximum value for a given $a$. Figure 5 shows $\Omega_{\mathrm{c}}$ in comparison with $\Omega_{\mathrm{R}}, \Omega_{\mathrm{V}}$ and $\Omega_{\mathrm{K}}$ versus $(1-a)$. It might be a chance coincidence but $\Omega_{\mathrm{c}}$ has the same value as $\Omega_{\mathrm{R}}$ for $a=0.99616$ at $r_{31}$, indicated by the lowest cross in Figure 5. It appears that there is a 1:1 resonance between $\Omega_{\mathrm{c}}$ and $\Omega_{\mathrm{R}}$, and this is the only "resonance" with $\Omega_{\mathrm{R}}, \Omega_{\mathrm{V}}$ and $\Omega_{\mathrm{K}}$ for $a<0.998$, which is the "Thorne" limit (Thorne 1974). In summary, $\Omega_{\mathrm{c}}=\left.2 \pi \frac{\partial v^{(\Phi)}}{\partial r}\right|_{\max }$ picks the same values for $a$ and $r$ as the parametric resonance model with commensurable Kepler orbits.

\section{Discussion}

The model predicts the mass of each of the four black hole objects with well estimated masses quite satisfactorily from measured HFQPOs. Nevertheless, there are a couple of interesting coincidences which I would like to point out and discuss.

It is interesting to note that the model based on parametric resonances between the vertical and radial epicyclic frequencies constrained by the requirement that any associated orbits should be commensurable orbits has only one solution, which selects just two orbits with radii $r_{31}$ and $r_{32}$, and restricts the angular momentum of the black hole to just one value of $a=a_{\mathrm{f}}=0.99616$. Even more interesting is the result that the orbital velocity $v^{(\Phi)}$ for the ZAMO changes its otherwise monotonical behaviour with radius and shows a local minimum and a local maximum which define a narrow radial annulus with $\partial v^{(\Phi)} / \partial r \geq 0$ for $a>0.9953=a_{\mathrm{c}}$. To my knowledge this feature of very rapidly rotating black holes has been overlooked so far. This certainly needs more study. Furthermore, the fact that $\Omega_{\mathrm{c}}=\left.2 \pi \frac{\partial v^{(\Phi)}}{\partial r}\right|_{\max }=\Omega_{\mathrm{R}}\left(r=r_{31}\right)$, suggests that there is a link between the "strange" behaviour of $v^{(\Phi)}$ and the radial epicyclic oscillation, such that $\Omega_{\mathrm{c}}\left(a_{\mathrm{f}}\right)=\Omega_{\mathrm{R}}\left(r_{31}, a_{\mathrm{f}}\right)$ is the condition to excite the radial and vertical epicyclic oscillations. I note that so far all this is produced in a single test particle environment.

The model predicts that there is just one single value of $a=a_{\mathrm{f}}$, and the HFQPOs of the three microquasars and the GC black hole are consistent with this. The same value of $a$ for four sources suggests that this is some sort of final state of $a$; if correct this would replace the former "Thorne" upper limit of $a=0.9982-0.9978$ (Thorne 1974). This would also imply that the excitation of the radial and vertical epicyclic oscillations at $r_{31}$ somehow regulate the angular momentum flow towards the black hole to an equilibrium point. $r_{31}$ is located in the ergosphere halfway between the horizon and the static limit; the distance $\delta r$ to the ergosphere limit is $\delta r=0.45$ in the equatorial plane. A radial oscillation with an amplitude greater than $\delta r$ would traverse the ergosphere boundary back and forth.

The oscillations at $r_{31}$ are likely to be powered by mass accretion and one could envisage the following scenario. At low mass accretion rates both the vertical and radial oscillations are stable and energy is stored in the corresponding wave; with increasing accretion rate the vertical oscillations become unstable because they are no longer capable of storing the energy and/or angular momentum but the energy is released in flares or bursts. I suggest that this is the state in which the GC black hole is. Observations show that the quiescent state $\mathrm{X}$-ray luminosity of $\mathrm{Sgr} \mathrm{A}^{*}$ is about 11 orders of magnitude below the Eddington limit (Baganoff et al. 2001; Porquet et al. 2003), which might indicate that the accretion rate is fairly low, although other mechanisms for the low luminosity have been proposed. The time scale for the rise time of the flare should be a half up to a few times of the period of the vertical oscillations, i.e. about 350 to $1000 \mathrm{~s}$, and the duration of such a flare should be about a few times of the period of the radial oscillations, i.e. 40 to $120 \mathrm{~min}$. These time-scales have been observed in the Chandra (Baganoff et al. 2001) flare and the XMM-Newton flare (Porquet et al. 2003; Aschenbach et al. 2004).

Recently Bower et al. (2004) have reported measurements by which $\operatorname{Sgr} \mathrm{A}^{*}$ has been spatially resolved at a wavelength of $7 \mathrm{~mm}$, and they estimate its size to be $<2.4 \times 10^{12} \mathrm{~cm}$ at a wavelength of $1.3 \mathrm{~mm}$ for a distance of $8 \mathrm{kpc}$. I note that the diameter of the region, where the vertical epicyclic mode is at work, is $2 \times r_{31}=1.5 \times 10^{12} \mathrm{~cm}$, whereas the diameter of the $r_{32}$ annulus is $3.8 \times 10^{12} \mathrm{~cm}$, which appears to be incompatible with the wavelength extrapolated radio measurements, if the X-ray emitting region is not more extended than the radio source. This result seems to confirm that the $\mathrm{Sgr}$ A* emission region is restricted to an area with a radius $r \leq r_{31}$. 
At even greater accretion rates there are no stable vertical oscillations any more, the $3: 1$ resonance disappears, and probably a significant fraction of the energy of the accreting matter is lost in vertical direction in a non-periodic fashion or stored in the radial mode, the amplitude of which will grow. Eventually the radial wave will reach $r_{32}$ and power the vertical and radial modes at $r_{32}$ because of their parametric "cross" resonances (Sect. 2). This is the 3:2 state I suggest the three microquasars are in. The X-ray luminosities of the three microquasars are very high, close to or possibly slightly above the Eddington limit. The accretion rate is certainly fairly high. Eventually, outbursts may also occur in the 3:2 state, when the vertical mode oscillations become unstable temporarily. Of course, a quantitative relation between oscillation state and accretion rate is still to be worked out.

The three microquasars are known to have jets, and Bower et al. (2004) state that their radio measurements of Sgr A* are consistent with jet models, so that all four sources might have jets. Therefore one might ask the question, whether the existence of any one of the following properties implies the existence of the other two: twin or triple HFQPOs, $a=a_{\mathrm{f}}$ and existence of a jet. For instance, has an accreting black hole with a jet $a=a_{\mathrm{f}}$ and HFQPOs? If so, it could be profitable to search for instance the extragalactic jet sources, make an estimate of the HFQPOs from Eqs. (4) and (5), and when found determine the black hole mass to percentage accuracy. A final speculative idea is the following. Is the instability of the vertical epicyclic oscillation at $r=r_{31}$ the seed for the creating of accretion driven black hole jets? The decrease of $v^{(\Phi)}$ of about $2 \%$ between $r_{\max }$ and $r_{\min }$ would correspond to an apparent loss of orbital kinetic energy of about $4.3 \mathrm{MeV} /$ nucleon in non-relativistic Newtonian approximation. Whether and how this energy can be made use of is unclear.

\section{Conclusion}

I have constructed a model built upon parametric $3: 1$ and 3:2 resonances between the vertical and radial epicyclic oscillations of a test particle orbiting a rotating black hole. If these two orbits are required to be commensurable orbits as far as their Keplerian frequencies are concerned there exists only one solution, which specifies uniquely the orbit radius $r_{31}=1.546$ of the $3: 1$ resonance, the orbit radius $r_{32}=3.919$ of the 3:2 resonance and the angular momentum $a_{\mathrm{f}}=0.99616$. The analysis of the general relativistic expression for the orbital velocity $v^{(\Phi)}$ in the ZAMO frame shows a deviation from the monotonic relationship between $v^{(\Phi)}$ and $r$ for $a>0.9953=a_{\mathrm{c}}$. $\partial v^{(\Phi)} / \partial r$ changes its sign over a narrow radial annulus, and there $\Omega_{\mathrm{c}}=2 \pi \frac{\partial v^{(\Phi)}}{\partial r} I_{\max }=\Omega_{\mathrm{R}}$. It seems that this condition is responsible for the excitation of radial and vertical epicyclic oscillations. The effect of $\partial v^{(\Phi)} / \partial r$ changing its sign is likely to be related to energy and angular momentum transfer via vertical and radial waves. This effect seems to have been overlooked in the past and needs more study. Since so far the model is based on test particle motion the entire effect is due to general relativity and for rapidly rotating black holes. It is not a collective effect. This, in some sense, consistent model predicts a new upper limit of $a=a_{\mathrm{f}}=0.99616$, which is slightly lower than the
"Thorne" limit (Thorne 1974). The model predicts the mass of fully spun-up black holes on the basis of measured twin (3:2) or triple (3:1) HFQPOs to the accuracy of the frequency measurements. It is suggested that the 3:1 resonance is active at low accretion rates, and the 3:2 resonance can be observed at very high accretion rates. At intermediate accretion rates $\Omega_{\mathrm{R}}\left(r=r_{31}\right)$ may be the only persistent frequency. The comparison of the masses derived with this model, on the basis of the measured HFQPOs, with the masses derived from dynamical measurements, i.e. binary orbit characteristics, shows an excellent agreement for the three microquasars GRO J1655-40, XTE J1550-564 and GRS 1915+105 and lowers the mass uncertainty significantly. The frequencies measured recently by Aschenbach et al. (2004) for Sgr A* appear to be arranged in a 3:2:1 ratio and the derived mass, which is 5.7 orders of magnitude higher than the lowest mass of the microquasars involved, agrees quite well with the dynamical mass at a much reduced uncertainty.

Acknowledgements. I would like to thank Joachim Trümper for inspiring discussions.

\section{References}

Abramowicz, M. A., \& Kluźniak, W. 2001, A\&A, 374, L19

Abramowicz, M. A., \& Kluźniak, W. 2002

[arXiv:astro-ph/0203314]

Abramowicz, M. A., \& Kluźniak, W. 2003, Interpreting Black Hole QPOs, Workshop on X-ray Timing (Boston: Rossi and Beyond) [arXiv:astro-ph/0312396]

Abramowicz, M. A., Almergren, G. J. E., Kluźniak, W., Thampan, A. V., \& Wallinder, F. 2002, CQGra, 19, L57

Abramowicz, M. A., Karas, V., Kluźniak, Lee, W. H., \& Rebusco, P. 2003, PASJ, 55, 467

Abramowicz, M. A., Kluźniak, W., Stuchlík, Z., \& Török, G. 2004a [arXiv: astro-ph/0401464]

Abramowicz, M. A., Kluźniak, W., McClintock, J. E., \& Remillard, R. A. 2004b, ApJ, 609, L63

Aschenbach, B., Grosso, N., Porquet, D., \& Predehl, P. 2004, A\&A, 417, 71

Baganoff, F. K., Bautz, M. W., Brandt, W. N., et al. 2001, Nature, 413, 45

Bardeen, J. M., Press, W. H., \& Teukolsky, S. A. 1972, ApJ, 178, 347

Boyer, R. H., \& Lindquist, R. W. 1967, J. Math. Phys., 8, 265

Bower, G. C., Falcke, H., Herrnstein, R. M., et al. 2004, Science, 304, 704

Brocksopp, C., Fender, R. P., McCollough, M., et al. 2002, MNRAS, 331,765

Cui, W., Shrader, C. R., Haswell, C. A., \& Hynes, R. I. 2002, ApJ, 535, L123

Eisenhauer, F., Schödel, R., Genzel, R., et al. 2003, ApJ, 597, L121

Filippenko, A. V., \& Chornock, R. 2001, IAU Circ., 7644

Genzel, R., Schödel, R., Ott. T., et al. 2003, Nature, 425, 934

Ghez, A. M., Duchêne, G., Matthews, K., et al. 2003, ApJ, 586, L127

Greene, J., Bailyn, C. D., \& Orosz, J. A. 2001, ApJ, 554, 1290

Greiner, J., Cuby, J. G., \& McCaughrean, M. J. 2001, Nature, 414, 522

Harlaftis, E. T., \& Greiner, J. 2004, A\&A, 414, L13

Kaiser, C. R., Gunn, K. F., Brocksopp, C., \& Sokoloski, J. L. 2004, ApJ, 612, 332

Kluźniak, W., \& Abramowicz, M. A. 2001a

[arXiv:astro-ph/0105057] 
Kluźniak, W., \& Abramowicz, M. A. 2001b, Acta Physica Polonica B, 32,3605

Kluźniak, W., \& Abramowicz, M. A. 2003, Proc. of the 12th Workshop on General Relativity, Tokyo 2002, ed. M. Shibata et al., 69 [arXiv: astro-ph/0304345]

Kluźniak, W., Abramowicz, M. A., \& Lee, W. H. 2003, Interpreting Black Hole QPOs, Workshop on X-ray Timing (Boston: Rossi and Beyond) [arXiv: astro-ph/0402013]

McClintock, J. E., \& Remillard, R. A. 2004 [arXiv: astro-ph/0306213]

Merloni, A., Vietri, M., Stella, L., \& Bini, D. 1999, MNRAS, 304, 155

Miller, J. M., Wijnands, R., Homan, J., et al. 2001, ApJ, 563, 928

Müller, A., \& Camenzind, M. 2004, A\&A, 413, 861

Nowak, M. A., \& Lehr, D. E. 1998, in Theory of Black Hole Accretion Discs (Cambridge University Press), ed. M. A. Abramowicz, G. Björnsson, \& J. E. Pringle, 233

Orosz, J. A., \& Bailyn, C. D. 1997, ApJ, 477, 876

Orosz, J. A., Groot, P. J., van der Klis, M., et al. 2002a, ApJ, 568, 845
Orosz, J. A., Polisensky, E. J., Bailyn, C. D., et al. 2002b, BAAS, 201, 1511

Porquet, D., Predehl, P., Aschenbach, B., et al. 2003, A\&A, 407, L17

Rebusco, P. 2004 [arXiv: astro-ph/0403341]

Remillard, R. A., Morgan, E. H., McClintock, J. E., Bailyn, C. D., \& Orosz, J. A. 1999, ApJ, 522, 397

Remillard, R. A., Muno, M. P., McClintock, J. E., \& Orosz, J. A. 2002, ApJ, 580, 1030

Remillard, R. A., Muno, M. P., McClintock, J. E., \& Orosz, J. A. 2003, A\&AS meeting \#35, \#30.03

Schnittman, J. D., \& Bertschinger, E. 2004, ApJ, 606, 1098

Schödel, R., Ott, T., Genzel, R., et al. 2002, Nature, 419, 694

Shapiro, S. L., \& Teukolsky, S. A. 1983, Black Holes, White Dwarfs, and Neutron Stars, The physics of compact objects (John Wiley \& Sons, Inc.)

Strohmayer, T. E. 2001a, ApJ, 552, L49

Stromayer, T. E. 2001b, ApJ, 554, L169

Thorne, K. S. 1974, ApJ, 191, 507 